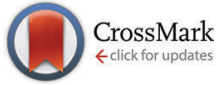

Cite this: Phys. Chem. Chem. Phys., 2015, 17, 1918

Received 5th June 2014, Accepted 26th November 2014

DOI: $10.1039 / c 4 c p 02482 f$

www.rsc.org/pccp

\section{Charge transfer to solvent dynamics in iodide aqueous solution studied at ionization threshold $\dagger$}

\author{
Alexander Kothe, ${ }^{a}$ Martin Wilke, ${ }^{a}$ Alexandre Moguilevski, ${ }^{a}$ Nicholas Engel, ${ }^{a}$ \\ Bernd Winter, ${ }^{a}$ Igor Yu. Kiyan ${ }^{\star a}$ and Emad F. Aziz ${ }^{* a b c}$
}

\begin{abstract}
We explore the early-time electronic relaxation in Nal aqueous solution exposed to a short UV laser pulse. Rather than initiating the charge transfer reaction by resonant photoexcitation of iodide, in the present timeresolved photoelectron spectroscopy study the charge-transfer-to-solvent (CTTS) states are populated via electronic excitation above the vacuum level. By analyzing the temporal evolution of electron yields from ionization of two transient species, assigned to CTTS and its first excited state, we determine both their ultrafast population and relaxation dynamics. Comparison with resonant-excitation studies shows that the highly excited initial states exhibit similar relaxation characteristics as found for resonant excitation. Implications for structure and dynamical response of the hydration cage are discussed.
\end{abstract}

\section{Introduction}

Electron-transfer photoreactions in aqueous and non-aqueous solutions play an important role in biology and solution chemistry, and how slow electrons cause damage of biological tissue is one particularly active research topic. ${ }^{1}$ Numerous studies have focused on characterizing the solvated electron and especially the hydrated electron, $\mathrm{e}^{-}$(aq) . These works can be typically divided into two main categories: ${ }^{2}$ the studies on the early-time dynamics and the mechanisms leading to $\mathrm{e}^{-}{ }_{(\mathrm{aq})}$, and the studies exploring the electronic properties of this species. The former is the topic of the present work, and the current understanding of $\mathrm{e}^{-}{ }_{\text {(aq) }}$ formation in aqueous solution will be briefly reviewed. A most recent account on the relaxation dynamics of photoexcited $\mathrm{e}^{-}$(aq) has been reported in ref. 3 .

Iodide in water is arguably the most common solution for studying either aspect, i.e. the formation of $\mathrm{e}^{-}{ }_{\text {(aq) }}$ and its properties. Since the atomic iodide anion has no vibrational degrees of freedom, the electron-transfer into solvent is fully characterized by the dynamical response of the water hydration shell. This facilitates interpretation of spectroscopic data.

\footnotetext{
${ }^{a}$ Joint Laboratory for Ultrafast Dynamics in Solutions and at Interfaces (JULiq), Institute of Methods for Material Development, Helmholtz-Zentrum Berlin, Albert-Einstein-Strasse 15, D-12489 Berlin, Germany.

E-mail: igor.kiyan@helmholtz-berlin.de,emad.aziz@helmholtz-berlin.de; Fax: +49 308062 14757; Tel: +49 30806212927

${ }^{b}$ Department of Physics, Freie Universität Berlin, Arnimallee 14, 14195 Berlin, Germany

${ }^{c}$ Institute for Molecular Science, Myodaiji, Okazaki 444-8585, Japan

$\dagger$ Electronic supplementary information (ESI) available: Fig. S1, 3D color map of the complete set of experimental transient spectra. See DOI: 10.1039/c4cp02482f
}

The electron-transfer reaction is triggered by photodetachment from iodide, and $\mathrm{e}^{-}{ }_{(\mathrm{aq})}$ is formed via population of short-lived intermediates. The exact nature of these transient states is not well understood though, as can be inferred from the quite different results obtained from time-resolved studies with the use of both transient photoabsorption and transient photoelectron (PE) spectroscopy. ${ }^{4-11}$ The method of PE spectroscopy is advantageous here since the actual electron binding energies of the species involved in the $\mathrm{e}^{-}$(aq) formation can be determined. It provides a direct signature of occurring transients, and thus allows for their explicit distinction. The initial step of the electron-transfer reaction is a charge transfer to the solvent which is attributed to the population of so called charge-transferto-solvent (CTTS) states. In most experiments this process was typically initiated by the resonant photoexcitation of a valence $5 p$ electron of $\mathrm{I}^{-}$(aq) into a configuration with an electron and an iodine atom being tight together. This structure constitutes a solvent-stabilized excited state, the CTTS state. In this CTTS configuration, the electron is bound in a potential well originating from the preexisting polarization of water dipoles oriented around the ion. Because of a large number of different hydration structures of iodide, already existing in the ground-state solution, the solvent cage rearrangement in the excited state leads to population of different CTTS states, with different energies. Although resonant excitation is indeed a crucial factor in previous experimental studies of the CTTS dynamics, the resonance condition is rather loosely defined since photon energies vary considerably in different experiments, typically between 5.1 and $6.2 \mathrm{eV}^{4,5,7,12}$ Importantly though, a significant fraction of the CTTS-state manifold, with energies fairly near the CTTS lowest state, is populated instantaneously by 1 -photon absorption. Relaxation of 
the CTTS states occurs within 100-500 fs. ${ }^{4,7,11}$ The observed spread in time constants is associated with aforementioned structure diversity. ${ }^{13}$

The immediate dissociation product of the CTTS state is thought to be a contact pair, ${ }^{11} \mathrm{I}^{0}: \mathrm{e}^{-}$, from which the solvated electron evolves directly within $\sim 1-2$ picoseconds. Other studies ${ }^{4,12}$ report two intermediates, $\mathrm{I}^{0}: \mathrm{e}^{-}$, and a solvent-separated pair, $\mathrm{I}^{0}-\mathrm{e}^{-}$. Yet another time-resolved PE spectroscopy study proposes the initial population of hot and cold short-lived intermediates. ${ }^{5}$ The difficulty in accurately assigning reaction transients is that their existence is inferred from kinetic modeling based on measured total electron yields or global absorption in a laser pump-probe experiment. Therefore, as was recently stressed, ${ }^{14}$ the direct spectroscopic signature of the intermediate states is yet to be provided.

In the time-resolved PE spectroscopy study of early-time electron dynamics in low-concentration NaI aqueous solution reported here, we explore the case of populating CTTS states via absorption of two photons of $4.65 \mathrm{eV}$ photon energy. This value is significantly smaller than the photon energies of $5.1 \mathrm{eV},^{12}$ $5.2 \mathrm{eV},{ }^{7} 5.5 \mathrm{eV},{ }^{4}$ and $6.2 \mathrm{eV},{ }^{5}$ applied in previous experiments to populate the CTTS state of iodide aqueous solution via direct 1-photon transition. Although the $4.65 \mathrm{eV}$ laser pulse may also lead to some direct population of the CTTS state ${ }^{13}$ (the threshold of the CTTS band lies at approximately $4.75 \mathrm{eV}$, ref. 8), the main excitation route in our study is absorption of two photons. This leads to the population of highly excited continuum states which are likely of CTTS character as well, with energies above the vacuum level. Here, the electron still remains close to the parent $\mathrm{I}^{0}{ }_{(\mathrm{aq})}$, within the size of the hydration shell, and electronic relaxation proceeds through population of low-lying CTTS states. In the transient PE spectra we identify both the lowest and the first excited states, CTTS and CTTS +1 . By following the temporal evolution of each spectral feature, recorded as a function of time delay between excitation and probe laser pulses, we quantify the ultrafast population and relaxation dynamics for each CTTS state individually. To our knowledge, formation of solvated electrons (and the initial population of CTTS states) via photodetachment into a state above the vacuum level has been only explored in one earlier work by inducing 3-photon absorption with the total excitation energy of $3 \hbar \omega=12 \mathrm{eV}^{15}$

\section{Experimental procedure}

We apply two-color time-resolved photoelectron spectroscopy to investigate the electron dynamics in a $20 \mathrm{mM} \mathrm{NaI}$ aqueous solution upon exposure to ultrashort UV laser pulses. In the pump-probe setup, the output of a femtosecond Ti:sapphire laser system was split to generate its third harmonic (TH), and to pump an optical parametric amplifier (OPA). The TH beam of $266 \mathrm{~nm}$ wavelength (4.65 eV photon energy), and the OPA output of $350 \mathrm{~nm}$ $(3.55 \mathrm{eV})$, were used to populate and to probe, respectively, the electronic states in the solution. The duration of both laser pulses was approximately $60 \mathrm{fs}$. Using an optical delay stage in the $\mathrm{TH}$ beam path, the time delay between the pump and the probe pulses could be varied with a precision of 1 fs.
In order to maintain high-vacuum conditions required for electron detection, the highly volatile aqueous solution was introduced into the interaction region as a liquid micro-jet. ${ }^{16}$ A quartz nozzle of $24 \mu \mathrm{m}$ diameter forms a fast (approximately $40 \mathrm{~m} \mathrm{~s}^{-1}$ ) jet with an initial laminar flow of a few millimeters length. A few centimeters downstream the flow, the sample was collected by a liquid trap, ${ }^{17}$ enabling to keep the residual gas pressure below $10^{-4}$ mbar in the interaction chamber during the experiment.

The two laser beams were focused onto the same spot within the laminar region of the liquid jet. Both focuses had approximately the same diameter of $20 \mu \mathrm{m}$, which is comparable to the jet diameter. The region of overlap was centered in front of the skimmer of a magnetic-bottle time-of-flight electron spectrometer. The skimmer size of $200 \mu \mathrm{m}$ ensured the gas pressure inside the spectrometer being below $10^{-6}$ mbar. In order to prevent saturation of the electron detector, the pulse energies of the pump and the probe laser beams were attenuated to 3 and $200 \mathrm{~nJ}$, respectively. At these conditions, less than one electron per laser pulse was recorded even though the pump and the probe pulses overlapped in time. The spectrometer characteristics, including the energy resolution and the electron collection efficiency, are presented in detail in ref. 18. Kinetic energies were calibrated by applying suitable retardation potentials to a grid positioned in front of the detector.

\section{Results and discussion}

\subsection{One-color spectra}

Before presenting the electron-emission spectra from the transient electronic states, we examine the photoelectron spectra obtained when using a single laser beam. Measured one-color spectra are shown in Fig. 1 (note the logarithmic scale of the signal intensity), where the top and the bottom panels display results for 4.65 and $3.55 \mathrm{eV}$ photon energy, respectively. The kinetic energy scale was corrected for the streaming potential associated with the friction of liquid flow in the nozzle capillary. With a correction value of $-0.2 \mathrm{eV}$, which is in good agreement with ref. 19, both onecolor spectra can be fully explained by contributions solely from ionization of the water $1 b_{1}, 3 a_{1}$ states, and from ionization of iodide into the $\mathrm{e}^{-}+\mathrm{I}^{0}\left({ }^{2} \mathrm{P}_{1 / 2}\right)$ and the $\mathrm{e}^{-}+\mathrm{I}^{0}\left({ }^{2} \mathrm{P}_{3 / 2}\right)$ continuum states associated with the formation of the iodine atom in the ${ }^{2} \mathrm{P}_{1 / 2}$ and ${ }^{2} \mathrm{P}_{3 / 2}$ spin-orbit states, respectively (we denote these contributions by $\mathrm{I}\left({ }^{2} \mathrm{P}_{1 / 2}\right)$ and $\left.\mathrm{I}\left({ }^{2} \mathrm{P}_{3 / 2}\right)\right)$. This is inferred from fitting a sum of Gaussian profiles, representing these contributions to the experimental spectra. The results of the fit are shown in Fig. 1, and the respective ionization channels are listed in Table 1. Individual Gaussian fits applied for each peak are in good agreement with widths and energy positions obtained from 1-photon ionization spectra of $\mathrm{I}^{-}(\mathrm{aq})$ solution using soft X-ray photon energies. ${ }^{20}$

Signal from the ground-state solvated electron (binding energy (BE) is $\sim 3.4-3.6 \mathrm{eV}^{5,6,21,22}$ ) is not apparent in our spectra because the laser pulse duration is shorter than the formation time of solvated electrons. Note that our interpretation of the 


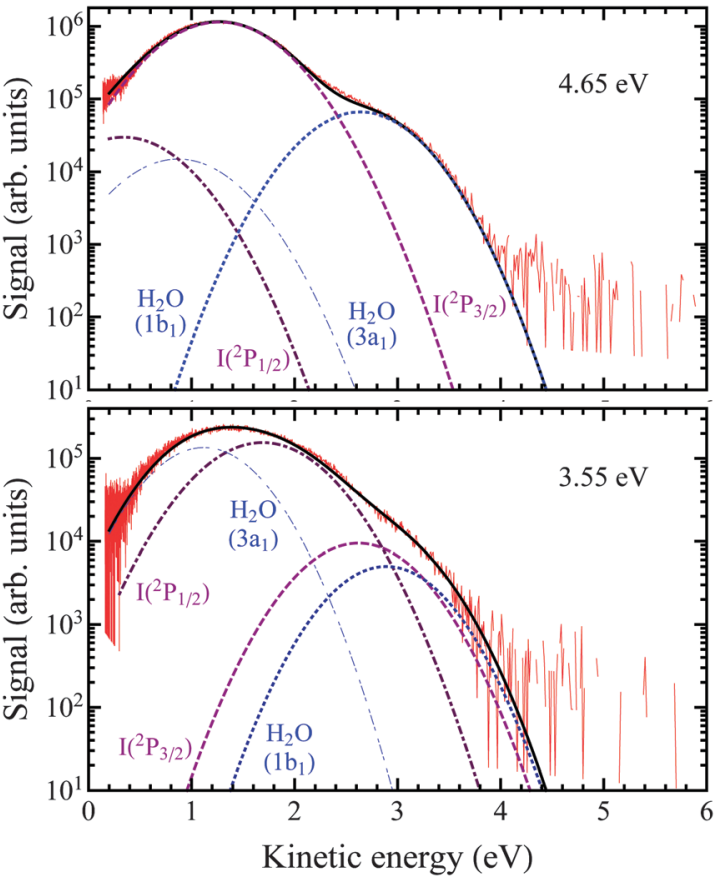

Fig. 1 One-color photoelectron spectra from $20 \mathrm{mM} \mathrm{Nal}$ aqueous solution obtained with the use of the pump ( $4.65 \mathrm{eV}$, top) and the probe ( $3.55 \mathrm{eV}$, bottom) beams. Black solid curves show the results of Gaussian fits. Contributions to the spectra arise from the states: $\mathrm{H}_{2} \mathrm{O}\left(3 \mathrm{a}_{1}\right)$ (blue, dash-dotted), $\mathrm{H}_{2} \mathrm{O}\left(1 \mathrm{~b}_{1}\right)$ (blue, dotted), I( $\left.{ }^{2} P_{1 / 2}\right)$ (purple, dash-dotted), and $I\left({ }^{2} P_{3 / 2}\right)$ (purple, dashed).

Table 1 Binding energies ${ }^{19}(\mathrm{BE})$ of the initial states of water and iodide, the number of absorbed photons of the pump beam $\left(N_{\text {pump }}\right)$ and of the probe beam $\left(N_{\text {probe }}\right)$, and the resulting kinetic energies $\left(E_{\text {kin }}\right)$ of photoelectrons

\begin{tabular}{llllll}
\hline State & $\mathrm{BE}(\mathrm{eV})$ & $N_{\text {pump }}$ & $E_{\text {kin }}(\mathrm{eV})$ & $N_{\text {probe }}$ & $E_{\text {kin }}(\mathrm{eV})$ \\
\hline $\mathrm{H}_{2} \mathrm{O}\left(3 \mathrm{a}_{1}\right)$ & 13.08 & 3 & 0.87 & 4 & 1.12 \\
$\mathrm{H}_{2} \mathrm{O}\left(1 \mathrm{~b}_{1}\right)$ & 11.31 & 3 & 2.64 & 4 & 2.89 \\
$\mathrm{I}\left({ }^{2} \mathrm{P}_{1 / 2}\right)$ & 8.96 & 2 & 0.34 & 3 & 1.69 \\
$\mathrm{I}\left({ }^{2} \mathrm{P}_{3 / 2}\right)$ & 8.03 & 2 & 1.27 & 3 & 2.62
\end{tabular}

photoelectron spectrum measured at $4.65 \mathrm{eV}$ photon energy is different from the one in ref. 5, where initial cold and hot states of the solvated electron have been postulated.

\subsection{Transient spectra}

Transient photoelectron spectra from the NaI aqueous solution were obtained by subtraction of the one-color distributions of Fig. 1 from the spectra recorded with both the pump $(4.65 \mathrm{eV})$ and the probe $(3.55 \mathrm{eV})$ pulses applied with a variable mutual time delay. The complete set of experimental transient spectra is presented in Fig. S1 of ESI. $\dagger$ Negative delay time corresponds to the probe pulse arriving first. In this case the transient spectra are featureless, implying that this laser beam does not populate transient states which could be probed by the $4.65 \mathrm{eV}$ beam. At positive time delays, the pump-probe photoelectron signal reveals two peaks at the kinetic energies (KE) of 1.03 and $1.48 \mathrm{eV}$ (see Fig. 2). A sum of two Gaussians is found to reproduce the experimental spectra. Given the $3.55 \mathrm{eV}$ photon energy of the ionizing probe pulse, the two peaks are assigned
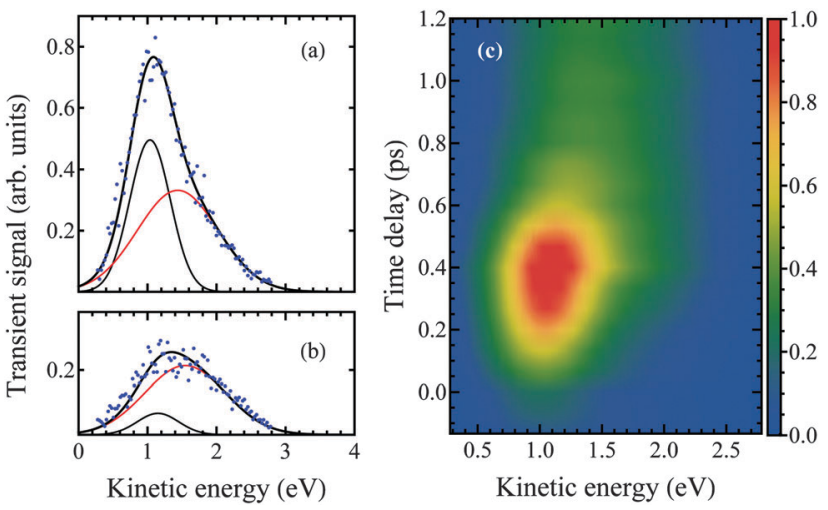

Fig. 2 Transient photoelectron signal. Panels (a) and (b) show two representative energy spectra recorded at the pump-probe time delay of 400 and $1200 \mathrm{fs}$, respectively. Dots (blue) represent the experimental data. Curves show individual contributions of two Gaussian peaks and their sum obtained from the fit. The temporal evolution of spectra, reconstructed from the Gaussian fits, is shown by the density plot in panel (c).

to transient species with BEs of 2.52 and $2.07 \mathrm{eV}$, respectively. The spectrum in Fig. 2(a) is measured at the time delay of $400 \mathrm{fs}$, at which instance the electron yield is maximal. In this spectrum the signal contribution of the low-KE peak is larger than for the high-KE peak. When increasing the time delay, the relative contributions eventually invert (see spectrum in Fig. 2(b)), qualitatively reflecting the different lifetimes of the involved transient states. A plot of the pump-probe PE signal reconstructed from the Gaussian fits to the raw spectra, as a function of both the kinetic energy and the time delay, yields the 3-dimensional contour map of Fig. 2(c). It is seen that both the 2.52 and $2.07 \mathrm{eV} \mathrm{BE}$ peaks remain at constant energy over the delay scan. Due to the small probe photon energy $(3.55 \mathrm{eV})$ applied in the present experiment, signal contributions from ionization of species evolving with yet higher binding energies cannot be accurately quantified.

The two transient peaks observed in the spectra are due to ionization of the CTTS and CTTS +1 states, respectively. Our assignment follows from the CTTS optical absorption characteristics in conjunction with the $\mathrm{I}^{-}{ }_{\text {(aq) }}$ electron binding energy. The CTTS absorption band from iodide aqueous solution exhibits a maximum at $5.52 \mathrm{eV}$ photon energy, ${ }^{8}$ and the absorption onset lies at $4.75 \mathrm{eV}$, i.e. above the $4.65 \mathrm{eV}$ photon energy of the pump beam used here. Electron binding energies of $\mathrm{I}^{-}{ }_{(\text {aq) }}$, attributed to the $\mathrm{I}\left({ }^{2} \mathrm{P}_{1 / 2}\right)$ and $\left.\mathrm{I}^{2} \mathrm{P}_{3 / 2}\right)$ parent states of the iodine atom, are 8.03 and $8.96 \mathrm{eV},{ }^{19}$ respectively. Subtracting the absorption band energy from these values, we find that the CTTS center energy lies at approximately $2.5 \mathrm{eV}$ below the vacuum threshold, which is in good agreement with our experimental value of $2.52 \mathrm{eV}$. An explicit experimental value of the CTTS +1 energy for iodide has not been reported but higher-lying states were discussed on the basis of quantummechanical computer simulations ${ }^{23}$ focusing on the branching between a direct photodetachment channel and adiabatic detachment. Our assignment of the $2.07 \mathrm{eV}$ peak to the CTTS + 1 state is based on a comparison with chloride experimental data (from soft-X-ray resonant Auger-electron spectroscopy ${ }^{24}$ ), 
where $1.2 \mathrm{eV}$ BE has been measured for the CTTS +1 state of chloride (aq), and $2.6 \mathrm{eV}$ for its lowest CTTS state. The same study also reports on higher-lying CTTS states, with energies of 0.4 and $1.9 \mathrm{eV}$ above the vacuum level. Such latter states play a crucial role in the present study, as we discuss below.

\subsection{Electron dynamics, kinetics, and modeling}

The pump-pulse photon energy of $4.65 \mathrm{eV}$ is not sufficient to populate the observed $\mathrm{I}^{-}$(aq) CTTS state, with a center BE of $2.52 \mathrm{eV}$, by 1-photon absorption (see Fig. 3(a and b) for an illustration of the energy levels). Absorption of a single photon rather populates the lower-energy tail of the CTTS band, as shown in the recent fluorescence study. ${ }^{13}$ To interpret our observations, one should thus consider 2-photon excitation, which is of resonance character due to the above mentioned one-photon transition to the low-energy side of the CTTS band. This inevitably implies the initial population of intermediate states with approximate energies of 0.3 and $1.3 \mathrm{eV}$ above the vacuum level (denoted by $\mathrm{X}_{1,2}$ in Fig. 3(a)). These energies are defined by the difference between the absorption energy of two photons and the binding energies of $\mathrm{I}^{-}{ }_{(\mathrm{aq})}$ attributed to the $\mathrm{I}\left({ }^{2} \mathrm{P}_{1 / 2}\right)$ and $\mathrm{I}\left({ }^{2} \mathrm{P}_{3 / 2}\right)$ states of the parent iodine atom, respectively. The same argument applies when considering the population of the CTTS + 1 state via absorption of two photons of the pump pulse. Note that the observation of the transient species at $1.48 \mathrm{eV}$ KE via absorption of an additional probe photon can be ruled out due to energy mismatch, and also electronically excited transient states in water have not been observed under comparable 2-photon excitation, in conjunction with a third probe photon. ${ }^{26}$ The continuum states are not observed in the transient spectra most probably because of a low ionization cross section of these states, and due to insufficient time resolution of the experiment. The exact nature of $\mathrm{X}_{1,2}$ is not known but it is likely of CTTS character as well, analogous to aforementioned findings in $\mathrm{Cl}^{-}{ }_{(\mathrm{aq})}{ }^{24}$

In order to determine the population and relaxation dynamics of the CTTS and CTTS +1 transient states observed in the photoelectron spectra, we examine the respective timedependent ionization yields displayed in Fig. 4. The yields were obtained by integration of the photoelectron signal for a given delay time. Since both transient peaks are sufficiently well separated in the energy spectra, the kinetics of electron population can be analyzed individually for each of the two spectral components. This makes modeling in principle more unique, as compared to fitting of the total (global) electron yield in which case important information remains unconsidered. On the other hand, our system is yet too complex for a complete and accurate description of the relaxation dynamics. The reason is that experiments, the one reported here and previous time-resolved PE or transient absorption studies, probe only a fraction of all possible channels, namely the ones leading to population of the CTTS states. Unless all competing relaxation channels, which can be of non-radiative or radiative character, are taken into account quantification of the electron dynamics is elusive. And yet, with the experimental information of Fig. 2 and 4 , we arrive at several crucial conclusions regarding the excitation and immediate de-excitation dynamics in $\mathrm{NaI}$ aqueous solution. This is achieved by developing a model that describes the observed temporal evolution of ionization yields in Fig. 4.

The main model ingredients are depicted in the combined energy-level - electronic-transition scheme of Fig. 3(b). For the sake of simplicity, we treat $\mathrm{X}_{1}$ and $\mathrm{X}_{2}$ as a single state, $\mathrm{X}$, which implies that both continuum states essentially populate the same lower-lying CTTS states. We obtain very similar results when assuming that CTTS +1 and CTTS states are decoupled or

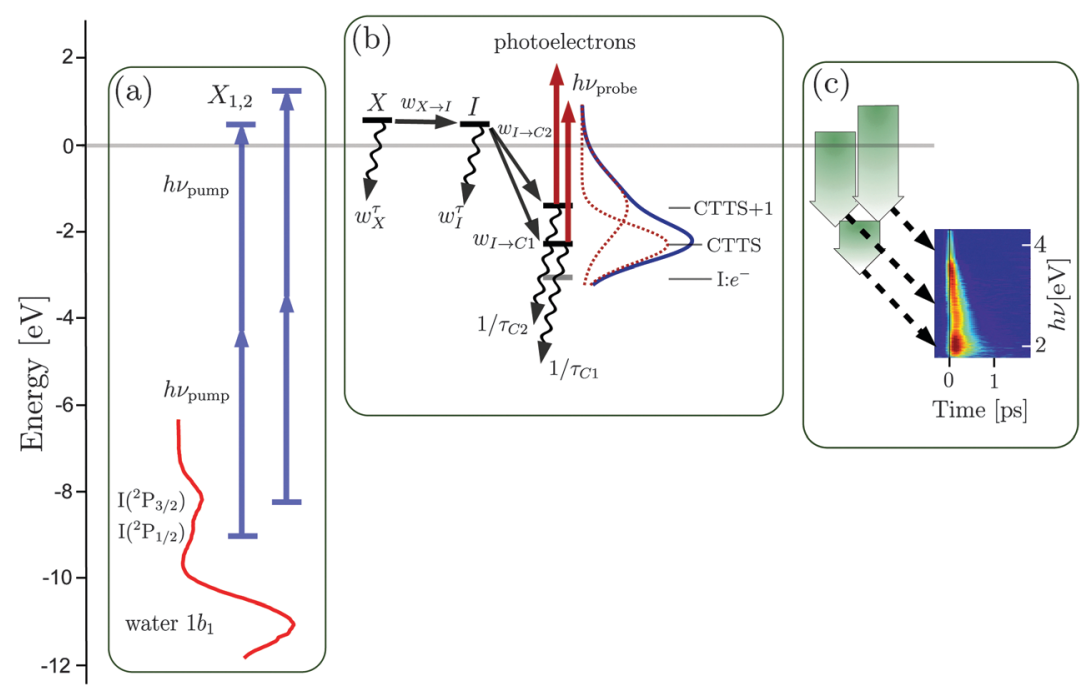

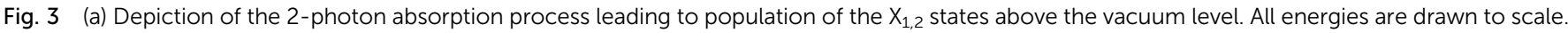

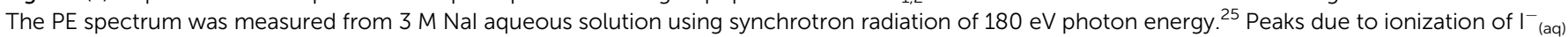

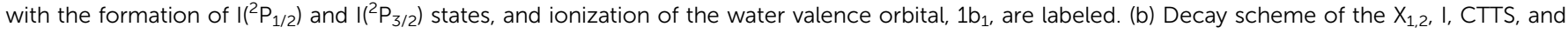

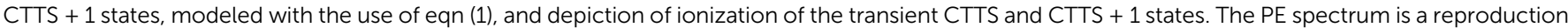

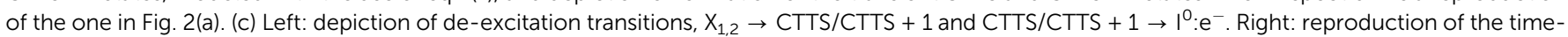
resolved fluorescence spectrum from ref. 13. The correspondence in energies with the present experiment is indicated by dashed arrows. 


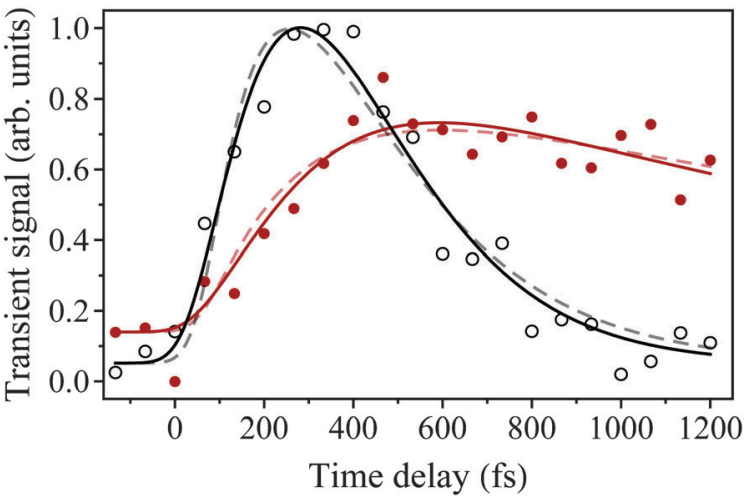

Fig. 4 Time dependency of the ionization yield from the transient states of $2.52 \mathrm{eV}$ (open circle) and $2.07 \mathrm{eV}$ (closed circles) binding energies. Solid curves represent results of the model described by eqn (1). Dashed curves show results of the model with the intermediate state I being excluded.

coupled (decay from CTTS +1 into CTTS is taken into account), respectively, as discussed in ref. 27 . In either case does successful modeling require a 2-step de-excitation of $\mathrm{X}_{1,2}$ via an intermediate state, denoted by I in Fig. 3(b). The need for introducing I will be discussed in detail below. Both the $\mathrm{X}$ and I states are also allowed to decay via other channels, not leading to population of the CTTS states, but their nature is not detailed here. Finally, we allow the CTTS and CTTS +1 state to decay. This leads to the following system of differential equations which we use to describe the experimentally observed temporal evolution of the electron yields:

$$
\begin{aligned}
& \dot{n}_{\mathrm{X}}=-\frac{1}{\tau_{\mathrm{X}}} n_{\mathrm{X}}+P(t), \\
& \dot{n}_{\mathrm{I}}=w_{\mathrm{X} \rightarrow \mathrm{I}} n_{\mathrm{X}}-\frac{1}{\tau_{\mathrm{I}}} n_{\mathrm{I}}, \\
& \dot{n}_{\mathrm{C}_{1}, \mathrm{C}_{2}}=w_{\mathrm{I} \rightarrow \mathrm{C}_{1}, \mathrm{C}_{2}} n_{\mathrm{I}}-\frac{1}{\tau_{\mathrm{C}_{1}, \mathrm{C}_{2}}} n_{\mathrm{C}_{1}, \mathrm{C}_{2}} .
\end{aligned}
$$

Here $n_{\mathrm{X}, \mathrm{I}, \mathrm{C}_{1}, \mathrm{C}_{2}}$ denote the populations of the X, I, CTTS, and CTTS + 1 states, respectively, $\tau_{\mathrm{X}, \mathrm{I}, \mathrm{C}_{1}, \mathrm{C}_{2}}$ represent their lifetimes, $w_{\mathrm{X} \rightarrow \mathrm{I}}, w_{\mathrm{I} \rightarrow \mathrm{C}_{1}}$, and $w_{\mathrm{I} \rightarrow \mathrm{C}_{2}}$ are the rates of the relaxation transitions depicted in Fig. 3(b), and $P(t)$ describes the time-dependent population rate of the $\mathrm{X}$ state with the actual temporal envelope of the pump pulse taken into account. The pulse shape is assumed to be Gaussian. Apart from the decay rates of the X and I states considered in eqn (1), other relaxation channels of these states are represented by the rates $w_{\mathrm{X}}^{\mathrm{r}}$ and $w_{\mathrm{I}}^{\mathrm{r}}$, respectively. As mentioned above, these decay channels can be radiative or non-radiative, the latter accounting e.g. for recombination of electron-hole pairs or by direct relaxation to the solvated electron state. The relation between the lifetimes and the decay rates of the $\mathrm{X}$ and I states can be expressed as

$$
\begin{aligned}
& \frac{1}{\tau_{\mathrm{X}}}=w_{\mathrm{X} \rightarrow \mathrm{I}}+w_{\mathrm{X}}^{\mathrm{r}}, \\
& \frac{1}{\tau_{\mathrm{I}}}=w_{\mathrm{I} \rightarrow \mathrm{C}_{1}}+w_{\mathrm{I} \rightarrow \mathrm{C}_{2}}+w_{\mathrm{I}}^{\mathrm{r}} .
\end{aligned}
$$

The system of differential equations in eqn (1) was solved numerically with the initial condition that all involved states were unpopulated at $t \rightarrow-\infty$. The ionization signal from the CTTS and CTTS +1 states was calculated by convolution of $n_{\mathrm{C}_{1}}(t)$ and $n_{\mathrm{C}_{2}}(t)$ with the temporal Gaussian envelope of the probe pulse, assuming that the probe step is non-saturated. The numerical results were fitted to the experimental data by means of a Nelder-Mead algorithm.

The numerical results are presented in Fig. 4 and show a good agreement with the experimental data. However, not all time constants of the transitions depicted in Fig. 3(b) can be unambiguously defined from the fit. In particular, the fit results are insensitive to the branching between $w_{\mathrm{X} \rightarrow \mathrm{I}}$ and $w_{\mathrm{X}}^{\mathrm{r}}$ rates for the decay of the $\mathrm{X}$ state. Only the lifetime $\tau_{\mathrm{X}}$ can be inferred from the fit as a robust parameter. This is also the case for characterization of the I state. The fit does however yield a robust value for the lifetime $\tau_{\mathrm{I}}$ but the decay branching into CTTS/CTTS + 1 states and other channels cannot be reliably determined. This restriction is directly related to the fact that we probe solely the populations of the CCTS and CTTS +1 states.

Inferred lifetimes are presented in Table 2 . We find that the decay time constants of the CTTS and CTTS +1 states are $\sim 130$ fs and $\sim 1.5 \mathrm{ps}$, respectively. Their decay products are not specified here. The time constant for the decay of the CTTS state derived here agrees well with the results from resonantexcitation experiments. ${ }^{28}$ This is perhaps not surprising when we assume the presence of a potential barrier for reaching the CTTS state. This barrier may be already small for transient states with energy of $\geq 1 \mathrm{eV}$ above the CTTS lowest state. The 1.5 ps time constant for the decay of the CTTS +1 state, which has not been reported before, appears to be surprisingly large. ${ }^{27}$ This might be due to a quite different structure motif of an iodide-electron pair in terms of the surrounding solvent cage arrangement, and may be related to the initial X-state hydration structure formed at large excess energy ( $>9 \mathrm{eV}$ photon absorption process). However, we have no experimental information that supports such a hypothesis and further investigations are needed to clarify this matter. We also do not exclude that the unexpected long lifetime of the $2.07 \mathrm{eV} \mathrm{BE}$ species might indicate that the broad width of this energy peak is not exclusively due to CTTS +1 photoemission.

We also applied a model, similar to the one described above, where we excluded the intermediate state I. The results of this model are also shown in Fig. 4. One can see that they reproduce the experimental data less satisfactory. In addition, we performed simulations where we allowed for both two-step and direct decay of the X state into the CTTS/CTTS +1 states. The respective fit to experimental data revealed that the two-step de-excitation rate is dominant. Therefore, we conclude that the

Table 2 Lifetimes of the X, I, CTTS, and CTTS + 1 states obtained from the modeling of the population and relaxation dynamics with the use of eqn (1), and from the modeling with the intermediate state I being excluded

\begin{tabular}{lllll}
\hline Model & $\tau_{\mathrm{X}}$ & $\tau_{\mathrm{I}}$ & $\tau_{\mathrm{C}_{1}}$ & $\tau_{\mathrm{C}_{2}}$ \\
\hline $\mathrm{X} \rightarrow \mathrm{I} \rightarrow \mathrm{C}_{1,2}$ & $150 \mathrm{fs}$ & $150 \mathrm{fs}$ & $130 \mathrm{fs}$ & $1.5 \mathrm{ps}$ \\
$\mathrm{X} \rightarrow \mathrm{C}_{1,2}$ & $220 \mathrm{fs}$ & - & $170 \mathrm{fs}$ & $2.0 \mathrm{ps}$
\end{tabular}


intermediate state I needs to be involved in the interpretation of experimental observations.

The nature of the intermediate state I can be inferred from the results of earlier studies on the capture of slow electrons in gaseous media. ${ }^{29}$ It was demonstrated that at high densities the three-body (electron and two neighboring molecules) collisions become probable, giving rise to a two-step process of nonradiative electron recombination via formation of a short-lived intermediate quantum system. This system represents a vibrationally excited molecular negative ion, which can be de-excited in a second collision step. Analogously, one could argue that the I state is representative of a $\mathrm{H}_{2} \mathrm{O}^{*}: \mathrm{e}^{-}$system, with the total energy matching the energy level of the $\mathrm{X}$ state. It is interesting to note that the exclusion of the I state in simulations results in a considerably longer lifetime of the X state (see Table 2). Both $\mathrm{X}$ and $\mathrm{I}$ states are then argued to represent different hydration structures of the same continuum state, and the structure rearrangement occurs on a time scale of approximately $100 \mathrm{fs}$.

Discussion of our experimental data would be incomplete without attempting to connect to the study of ref. 13 which uses the same photon energy, similar pulse energy, and only the NaI concentration is different, $1 \mathrm{M}$ versus $20 \mathrm{mM}$ in the present study. What is curious here is that the two studies are seemingly sensitive to entirely different excitation routes, and hence very different relaxation channels are probed in each case. Ref. 13 considers electronic excitation at the very onset of the CTTS absorption band, while the present work explores an indirect pathway, populating CTTS states through a continuum state. In our attempt to find out how these two studies possibly connect in terms of the information extracted (state assignment, relaxation mechanism) we noticed that the timedependent fluorescence appears to remarkably well match the processes presented in Fig. 3(b). Specifically, we find a good match between the emission wavelengths and the electron BEs, and in part even with the respective temporal characteristics. The former is best demonstrated with the help of Fig. 3(c) which depicts the principle transitions from the $\mathrm{X}_{1,2}$ states and from the lower-energy CTTS states. In the figure we also include the $\mathrm{e}^{-}$(aq) state $(\mathrm{BE} \sim 3.4 \mathrm{eV})$, which is approximately representative of $\mathrm{I}^{0}: \mathrm{e}^{-}$. Downward arrows represent the electronic decay transitions, and the arrow lengths correspond to actual energy differences. For example, $\mathrm{BE}_{\mathrm{X} 1}-\mathrm{BE}_{\mathrm{CTTS}}$ represents the largest energy difference of $\sim 3.8 \mathrm{eV}$ (center to center), and $\mathrm{BE}_{\mathrm{X} 2}-\mathrm{BE}_{\mathrm{CTTS}+1}$ has the smallest value of $\sim 2.4 \mathrm{eV}$. The lower arrow depicts the analogous energy release corresponding to the CTTS +1 CTTS $\rightarrow \mathrm{I}^{0}: \mathrm{e}^{-}$electronic transitions. Dashed cross-linking arrows mark the corresponding energy ranges in the fluorescence spectrum which is reproduced from ref. 13 at the right of Fig. 3(c). The incomplete correspondence to the lowest-energy transition of $\sim 1 \mathrm{eV}$ is of technical reason that caused a cutoff at $\sim 1.8 \mathrm{eV}(\sim 700 \mathrm{~nm})$ in the experimental fluorescence spectrum. ${ }^{13}$ At the present moment we have no satisfactory suggestion how to simultaneously interpret these two studies. We are convinced though that continuum transients have been involved in several previous works applying photon energies near the CTTS absorption onset.

\section{Conclusions}

Near-threshold ionization of NaI aqueous solution, which leads to the instantaneous population of highly excited states with energies above the vacuum threshold, is shown to be an efficient alternative route for populating CTTS states. These are the same states accessed by resonant photodetachment from iodide, and which are known to dissociate with a high probability for subsequent formation of the hydrated electron. Based on measured population times, our study indicates that the character of the initial solvent cage structures around the 2-photon excited iodide is similar to the case of 1-photon induced detachment. Also subsequent dynamical response of the water solvent is similar, as the large excess energy is rapidly dissipated via de-excitation. Further experiments are needed to detail our observations, including the exploration of nonradiative decay, and to quantify the importance of water multi-photon absorption as well as to characterize the dissociation products of the populated CTTS states.

\section{Acknowledgements}

This work was supported by the Helmholtz-Gemeinschaft via the VH-NG-635 grant and the extended investment of 2013 (E.F.A.) and the European Research Council grant No. 279344 (E.F.A.).

\section{References}

1 E. Alizadeh and L. Sanche, Chem. Rev., 2012, 112, 5578-5602.

2 L. Turi and P. J. Rossky, Chem. Rev., 2012, 112, 5641-5674.

3 M. H. Elkins, H. L. Williams, A. T. Shreve and D. M. Neumark, Science, 2013, 342, 1496-1499.

4 Y.-I. Suzuki, H. Shen, Y. Tang, N. Kurahashi, K. Sekiguchi, T. Mizunoc and T. Suzuki, Chem. Sci., 2011, 2, 1094-1102.

5 A. Lübcke, F. Buchner, N. Heine, I. V. Hertel and T. Schultz, Phys. Chem. Chem. Phys., 2010, 12, 14629-14634.

6 Y. Tang, H. Shen, K. Sekiguchi, N. Kurahashi, T. Mizuno, Y.-I. Suzuki and T. Suzuki, Phys. Chem. Chem. Phys., 2010, 12, 3653-3655.

7 F. Buchner, T. Schultz and A. Lübcke, Phys. Chem. Chem. Phys., 2012, 14, 5837-5842.

8 J. A. Kloepfer, V. H. Vilchiz, V. A. Lenchenkov and S. E. Bradforth, Chem. Phys. Lett., 1998, 298, 120-128.

9 J. A. Kloepfer, V. H. Vilchiz, V. A. Lenchenkov, X. Chen and S. E. Bradforth, J. Chem. Phys., 2002, 117, 776-778.

10 J. A. Kloepfer, V. H. Vilchiz, V. A. Lenchenkov, A. C. Germaine and S. E. Bradforth, J. Chem. Phys., 2000, 113, 6288-6307.

11 X. Chen and S. E. Bradforth, Annu. Rev. Phys. Chem., 2008, 59, 203-231.

12 H. Iglev, A. Trifonov, A. Thaller, I. Buchvarov, T. Fiebig and A. Laubereau, Chem. Phys. Lett., 2005, 403, 198-204.

13 F. Messina, O. Bräm, A. Cannizzo and M. Chergui, Nat. Commun., 2013, 4, 2119.

14 V.-T. Pham, T. J. Penfold, R. M. van der Veen, F. Lima, A. El Nahhas, S. L. Johnson, P. Beaud, R. Abela, C. Bressler, 
I. Tavernelli, C. J. Milne and M. Chergui, J. Am. Chem. Soc., 2011, 133, 12740-12748.

15 F. H. Long, X. Shi, H. Lu and K. B. Eisenthal, J. Phys. Chem., 1994, 98, 7252-7255.

16 B. Winter and M. Faubel, Chem. Rev., 2006, 106, 1176-1211.

17 K. M. Lange, A. Kothe and E. F. Aziz, Phys. Chem. Chem. Phys., 2012, 14, 5331-5338.

18 A. Kothe, J. Metje, M. Wilke, A. Moguilevski, N. Engel, R. AlObaidi, C. Richter, R. Golnak, I. Y. Kiyan and E. F. Aziz, Rev. Sci. Instrum., 2013, 84, 023106.

19 N. Kurahashi, S. Karashima, Y. Tang, T. Horio, B. Abulimiti, Y.-I. Suzuki, Y. Ogi, M. Oura and T. Suzuki, J. Chem. Phys., 2014, 140, 174506.

20 R. Weber, B. Winter, P. M. Schmidt, W. Widdra, I. V. Hertel, M. Dittmar and M. Faubel, J. Phys. Chem. B, 2004, 108, 4729-4736.

21 A. T. Shreve, T. A. Yen and D. M. Neumark, Chem. Phys. Lett., 2010, 493, 216-219.
22 K. R. Siefermann, Y. Liu, E. Lugovoy, O. Link, M. Faubel, U. Buck, B. Winter and B. Abel, Nat. Chem., 2010, 2, 274-279.

23 W. S. Sheu and P. J. Rossky, J. Phys. Chem., 1996, 100, 1295-1302.

24 B. Winter, E. F. Aziz, N. Ottosson, M. Faubel, N. Kosugi and I. V. Hertel, J. Am. Chem. Soc., 2008, 130, 7130-7138.

25 R. Seidel, S. Thuîrmer and B. Winter, J. Phys. Chem. Lett., 2011, 2, 633-641.

26 A. Hertwig, H. Hippler and A.-N. Unterreiner, Phys. Chem. Chem. Phys., 1999, 1, 5633-5642.

27 W.-S. Sheu and P. J. Rossky, Chem. Phys. Lett., 1993, 202, 186-190.

28 Y. Tang, Y.-I. Suzuki, H. Shen, K. Sekiguchi, N. Kurahashi, K. Nishizawa, P. Zuo and T. Suzuki, Chem. Phys. Lett., 2010, 494, 111-116.

29 L. M. Chanin, A. V. Phelps and M. A. Biondi, Phys. Rev., 1962, 128, 219-230. 